\title{
Application of Square-Root Unscented Kalman Filter Smoothing Algorithm in Tracking Underwater Target
}

\author{
Qiguo $\mathrm{YAO}^{\mathrm{a},{ }^{*}}$, Yuxiang $\mathrm{SU}^{\mathrm{b}}$, Lili $\mathrm{LI}^{\mathrm{c}}$ \\ School of Naval Architecture \& Mechanical-electrical Engineering, Zhejiang Ocean University, \\ Zhoushan, Zhejiang, 316022, China \\ ayaoqiguo@163.com, b'suyuxiang82@163.com, 'Cilili@zjou.edu.cn, ${ }^{*}$ corresponding author
}

\begin{abstract}
Keywords: Target Tracking, Square-root Unscented Kalman Filter, Smoothing Algorithm; Forward-filtering, Backward- smoothing
\end{abstract}

\begin{abstract}
In passive tracking, the nonlinearity may cause computational complication and precision degradation. To solve this problem, a novel filtering-smoothing algorithm based on Square-Root Unscented Kalman Filter (SR-UKFS) is proposed to track underwater target. In the SR-UKFS algorithm, the Square-Root Unscented Kalman Filter (SR-UKF) is used as forward-filtering algorithm to provide current location results, and the Rauch-Tung-Striebel (RTS) algorithm smoothes the previous state vector and covariance matrix using the current location results. Comparative analysis and validation are made on the tracking performances of SR-UKFS algorithm and SR-UKF algorithm, and the simulation results show that, under the same conditions, the SR-UKFS can more effectively improve the tracking precision than the SR-UKF algorithm. The SR-UKFS algorithm can reduce nearly $59 \%$ of the position error and nearly $54 \%$ of the velocity error.
\end{abstract}

\section{Introduction}

Using single observer to track targets avoids complex time synchronization. It provides significant practical value with strong independence and good motility. When relative movements exist between the observer and target and so does observability, the observer can track targets [1]. Underwater target motion analysis is a technique for estimation of target motion parameters, which is based on a series of measured data sequence (including azimuth angle and pitch angle) from hydrophone array of sonar platform [2]. The core of this technique is filtering algorithm, which is utilized to locate and track. Because of severe system nonlinearity, underwater target tracking shall face the linearization problems of state equation and measurement equation. Regarding the nonlinearity of state equation or measurement equation, related literatures adopt many modified Kalman filtering algorithms, like extend Kalman filter (EKF), UKF, SR-UKF and so on[3].

EKF property depends on partial nonlinear strength. During the linearization process of EKF, Jacobian matrix is required, which sometimes makes difficulty in realization [4]. UKF approximates to-be-estimated parameters by constructing a group of certain weighted sample points, which avoids the linearization modeling of nonlinear objects and calculation of Jacobian matrix as a filtering algorithm which can be directly utilized in nonlinearity system for mode estimation[5]. But in practical utilization, the data round-off error may make covariance matrix negative definite, which fails the UKF algorithm in calculating the matrix square root. Compared with EKF and without additional calculated amount, UKF makes it that estimated accuracy and rate of convergence are apparently enhanced. SR-UKF algorithm estimates square-rooting matrix of error to do the recursive calculation, which solves the problem of negative definite covariance matrix and filtering divergence due to calculation error and noise signal in standard UKF algorithm, and enhances the accuracy and stability of filtering[6].

RTS smoothing algorithm is a fixed interval optimal smoothing technique, it greatly simplifies the process of calculation. Smoothing can build a coherent connection at series of estimations, which makes the estimations more resistant to disturbance [7].

Based on SR-UKF, SR-UKFS adds backward smoothing for better accuracy of target state estimation in last moment, and enhances target state estimation accuracy of this moment [8]. 
According to the relative location of target and observation platform, the paper is based on the information of noise measurement of azimuth angle and pitch angle from passive sonar, tracks the course of underwater target with the utilization of SR-UKFS, and makes the simulation comparisons between standard UKF algorithm and SR-UKF algorithm.

\section{Tracking Principle and System Model}

The basic function of passive sonar is to find direction to measure azimuth information (including azimuth angle and angular altitude) formed by wave beam. Azimuth angle includes the location information of target horizontal direction. Angular altitude includes the information of target depth. Among the research about orientation and tracking problem of underwater target, the shape and size of observer and target can be ignored as particle of space, and the acceleration of target can be seen as a result of noise excitation and a Gaussian random process[9].

In passive sonar tracking system, the information of sonar measurement can be obtained from a spherical coordinate [10]. Target dynamic model is usually constructed in rectangular coordinate system. Thus, the target tracking of sonar becomes a nonlinear estimation problem. The main methods to solve this problem are EKF, UKF and SR-UKF. This paper adopts SR-UKFS algorithm to track the target, namely, firstly uses SR-UKF algorithm to estimate target state, and then uses RTS smoothing algorithm to obtain the target state estimation of last moment, and finally adopts SR-UKF algorithm to estimate the target state of this moment.

A linear dynamic model and a nonlinear observing model can be used to establish a target motion model in rectangular coordinate. The state variable is $X(k)=\left[x(k), y(k), z(k), v_{x}(k), v_{y}(k), v_{z}(k)\right]^{T}$, where $x, y, z$ means the relative location of $X, Y, Z$ directions, $v$ means the relative speed of $X, Y, Z$ directions. The discrete state equation of system is

$$
X(k+1)=\Phi(k+1, k) X(k)+\Gamma(k) U(k)
$$

Where,

$$
\Phi(k+1, k)=\left[\begin{array}{cccccc}
1 & 0 & 0 & T & 0 & 0 \\
0 & 1 & 0 & 0 & T & 0 \\
0 & 0 & 1 & 0 & 0 & T \\
0 & 0 & 0 & 1 & 0 & 0 \\
0 & 0 & 0 & 0 & 1 & 0 \\
0 & 0 & 0 & 0 & 0 & 1
\end{array}\right], \square(k)=\left[\begin{array}{ccc}
T^{2} / 2 & 0 & 0 \\
0 & T^{2} / 2 & 0 \\
0 & 0 & T^{2} / 2 \\
T & 0 & 0 \\
0 & T & 0 \\
0 & 0 & T
\end{array}\right]
$$

$\Phi(k+1, k)$ means state transition matrix; $\Gamma(k)$ is the matrix of system noise, $U(k)=\left[u_{x}(k), u_{y}(k), u_{z}(k)\right]^{T}$ is the systematic process noise caused by target acceleration, process noise is zero-mean Gaussian white noise, its variance matrix is $Q=\operatorname{diag}\left(\left[\sigma_{u_{x}}, \sigma_{u_{y}}, \sigma_{u_{z}}\right]\right)$. T is the sampling period of system.

The observed quantity of system includes azimuth angle $\theta(k)$ and pitch angle $\varphi(k)$. In rectangular coordinate system, the formula of azimuth angle and pitch angle can be seen in formula (2):

$$
\left[\begin{array}{c}
\theta(k) \\
\varphi(k)
\end{array}\right]=\left[\begin{array}{l}
\arctan (x(k) / y(k)) \\
\arctan \left(z(k) / \sqrt{x(k)^{2}+y(k)^{2}}\right.
\end{array}\right]
$$

When the target depth $z(k)$ is a fixed value, the observed quantity of azimuth angle and pitch angle can be transferred to the position quantity of target indirection $X$ and $Y$, and the transition measurement equation of system can be seen in formula (3):

$$
Z(k)=H(X(k))=\left[\begin{array}{c}
x(k) \\
y(k)
\end{array}\right]=\left[\begin{array}{l}
z(k) /\left(\tan (\varphi(k)) \sqrt{1+\tan ^{2}(\theta(k))}\right) \\
z(k) \tan (\theta(k)) /\left(\tan (\varphi(k)) \sqrt{1+\tan ^{2}(\theta(k))}\right)
\end{array}\right]+V(k)
$$


The measuring error of azimuth angle and pitch angle is relatively independent zero-mean Gaussian white noise, $V(k)=\left[v_{\theta}(k), v_{\varphi}(k)\right]^{T}$ is the measurement noise caused by azimuth angle and pitch angle, its variance matrix is $R$, and $R=\operatorname{diag}\left(\left[\sigma_{\theta}, \sigma_{\varphi}\right]\right)$.

\section{SR-UKFS Algorithm}

UKF is proposed by Julier, and widely used in the field of nonlinear estimation. But in practice, due to the round-off error in numerical calculation, sometimes we may get negative definite covariance matrix, which leads to the stoppage of UKF filter [11]. In order to avoid failure, SR-UKF uses covariance square root to replace the covariance to take part in recursive calculation, which ensures the half positive definitiveness of covariance in basic state has better numerical haracteristics.

In the process of UKF filtering, the calculation of new sigma point in every update requires a considerable number of calculations. Every time we must calculate the square root of state covariance matrix $P$, and assumes $S S^{T}=P$. In the process of SR-UKF filtering, $S$ will be recorded to avoid heavy decomposition calculation in every sampling, which enhances the operation speed of UKF. $Q R$ disintegration and Cholesky disintegration update are two important concepts in SR-UKF.

$Q R$ disintegration: for matrix $A \in R^{L \times N}(N \geq L)$, finding an orthogonal matrix $Q \in R^{N \times N}$ and an upper triangular matrix $R \in R^{N \times L}$ to make $A^{T}=Q R$, which is doing a $Q R$ disintegration for matrix $A$. $\operatorname{qr}\{\cdot\}$ can be used as $Q R$ disintegration with $R$ as returned value. According to the analysis knowledge of matrix, $R^{T}=S=\operatorname{chol}(P), R$ is also the transposition of Cholesky coefficient $S$ in matrix $P=A A^{T}$.

Cholesky disintegration update: if $S$ is the Cholesky disintegration of matrix $P=A A^{T}$, and $S=\operatorname{chol}(P)$, so the Cholesky disintegration update of matrix $P \pm \sqrt{v} u u^{T}$ can be marked as $S=$ cholupdate $\{S, u, \pm v\} . u$ usually is a column of vectors, but if $u$ is a matrix which includes factors in $M$ column, and uses vectors in $M$ column to $M$ times first order Cholesky update successively. In filtering process, $S$ displaces $P$ to participate in recursive calculation which can ensure the nonnegative definitiveness of covariance matrix for effective filtering[12].

For both UKF algorithm and SR-UKF algorithm, target state estimation of this moment is related to both current measured value and the state estimation of last moment. Thus, the state estimation accuracy enhancement of last moment can enhance the tracking accuracy.

SR-UKFS uses RTS algorithm as the forward filter algorithm to backward smooth the received target state estimation, to obtain a precise target state estimation of last moment, and uses SR-UKF for secondary filtering [14].

The calculation step of SR-UKFS is given as:

Step 1: Parameters initialize

$$
\begin{aligned}
& \hat{x}_{0}=E\left[x_{0}\right] \\
& P_{0}=E\left[\left(x_{0}-\hat{x}_{0}\right)\left(x_{0}-\hat{x}_{0}\right)^{T}\right] \\
& S_{0}=\operatorname{chol}\left(P_{0}\right)
\end{aligned}
$$

Step 2: Selection of sigma points

$$
\chi_{k-1}=\left[\hat{x}_{k-1} \hat{X}_{k-1}+\sqrt{n+\kappa} S_{k} \hat{x}_{k-1}-\sqrt{n+\kappa} S_{k}\right]
$$

Step 3: Time update equations

$$
\begin{aligned}
& \chi_{k \mid k-1}^{*}=\Phi_{k \mid k-1} \chi_{k-1} \\
& \hat{X}_{k}^{-}=\sum_{i=0}^{2 n} W_{i}^{(m)} \chi_{i, k \mid k-1}^{*} \\
& \hat{S}_{k}^{-}=\operatorname{qr}\left(\left[\sqrt{W_{1}^{c}}\left(\chi_{1: 2 n, k \mid k-1}^{*}-\hat{X}_{k}^{-}\right) \sqrt{\Gamma Q \Gamma^{T}}\right]\right)
\end{aligned}
$$




$$
\begin{aligned}
& S_{k}^{-}=\operatorname{cholupdate}\left(\hat{S}_{k}^{-}, \chi_{0, k}^{*}-\hat{\chi}_{k}^{-}, W_{0}^{(c)}\right) \\
& \chi_{k \mid k-1}=\left[\hat{X}_{k}^{-} \hat{X}_{k}^{-}+\sqrt{n+\kappa} S_{k}^{-} \hat{X}_{k}^{-}-\sqrt{n+\kappa} S_{k}^{-}\right] \\
& Z_{k \mid k-1}=H\left(\chi_{k \mid k-1}\right) \\
& \hat{Z}_{k}^{-}=\sum_{i=0}^{2 n} W_{i}^{(m)} Z_{i, k \mid k-1}
\end{aligned}
$$

Step 4: Measurement update equations

$\hat{S}_{\bar{z}_{k}}=\operatorname{qr}\left(\left[\sqrt{W_{1}^{(c)}}\left[Z_{1: 2 n, k \mid k-1}-\hat{Z}_{k}^{-}\right] \sqrt{R}\right]\right)$

$$
S_{\bar{Z}_{k}}=\operatorname{cholupdate}\left(\hat{S}_{\bar{z}_{k}}, Z_{0, k}-\hat{Z}_{k}^{-}, W_{0}^{(c)}\right)
$$$$
P_{x_{k} Z_{k}}=\sum_{i=0}^{2 n} W_{i}^{(c)}\left[\chi_{i, k \mid k-1}-\hat{X}_{k}^{-}\right]\left[Z_{i, k \mid k-1}-\hat{Z}_{k}^{-}\right]^{T}
$$$$
K_{k}=\left(P_{x_{k} Z_{k}} / S_{\bar{Z}_{k}}^{T}\right) / S_{\bar{Z}_{k}}
$$$$
\hat{X}_{k}=\hat{X}_{k}^{-}+K_{k}\left(Z_{k}-\hat{Z}_{k}^{-}\right)
$$$$
U=K_{k} S_{\bar{Z}_{k}}
$$

$S_{k}=\operatorname{cholupdate}\left(S_{k}^{-}, U,-1\right)$

Step 5: Smoothing process

$$
\begin{aligned}
& \hat{x}_{k-1 \mid k}=\hat{x}_{k-1}+D\left(\hat{x}_{k-1}-\chi_{k \mid k-1}^{*}\right) \\
& S_{k-1 \mid k}=S_{k-1}+D\left(S_{k-1}-S_{k \mid k-1}\right) D^{T} \\
& D=S_{k-1} \Phi_{k}\left(S_{k \mid k-1}\right)^{-1}
\end{aligned}
$$

Step 6: Second forward SR-UKF filtering

Replacing $\hat{x}_{k-1}, S_{k-1}$ by $\hat{x}_{k-1 \mid k}, S_{k-1 \mid k}$ and repeating step 2 to 4 .

Where,

$$
\begin{aligned}
& W_{0}^{(m)}=\lambda /(n+\lambda), \\
& W_{0}^{(c)}=\lambda /(n+\lambda)+\left(1-\alpha^{2}+\beta\right), \\
& W_{i}^{(m)}=W_{i}^{(c)}=1 /[2(n+\lambda)] \quad(i=1,2, \cdots, 2 n)
\end{aligned}
$$

Where $\lambda=\alpha^{2}(n+\kappa)-n$ is scale parameter, $n$ is the dimension of state vector of system, $\alpha$ decides the degree of dispersion of sigma and usually sets as a small positive number, and $0.001<\alpha \leq 1$. $\kappa$ is another scale adjustment parameter, which is usually set as $0 . \beta$ includes the prior knowledge of $x$ probability distribution. For random variables which follow the Gaussian distribution, $\beta=2$ is the best situation.

\section{Simulation Results and Analysis}

During the observation, sonar observation platform is motionless. The relations of relative location constitute the three-dimensional situation with known depth. And $z=45 \mathrm{~m}, v_{z}=u_{z}=0$, the target state vector can be simplified as $X(k)=\left[x(k), y(k), v_{x}(k), v_{y}(k)\right]^{T}$. The simulation condition and related parameters: sampling period $T=0.1 \mathrm{~s}$, noise variance matrix of system $Q=\operatorname{diag}([1,1])$, the measured noise standard deviation of azimuth angle and pitch angle in passive sonar $\sigma_{\theta}=0.1 \mathrm{rad}$, 
$\sigma_{\varphi}=0.1 \mathrm{rad}$. The initial state vector of target is $X(0 \mid 0)=(250,433,-4,-6.9)^{T}$, the covariance matrix of original state error $P=\operatorname{diag}([50,50,5,5])$, the simulation time is $50 \mathrm{~s}$. The filtering parameter of SR-UKFS is set as $\alpha=0.002, \beta=2, \kappa=0$.

Under above-mentioned conditions, the author evaluates the performances of UKF, SR-UKF and SR-UKFS, which are used for underwater pure orientation targets.Fig. 1 is the comparison chart of the three kinds of algorithms in tracking.

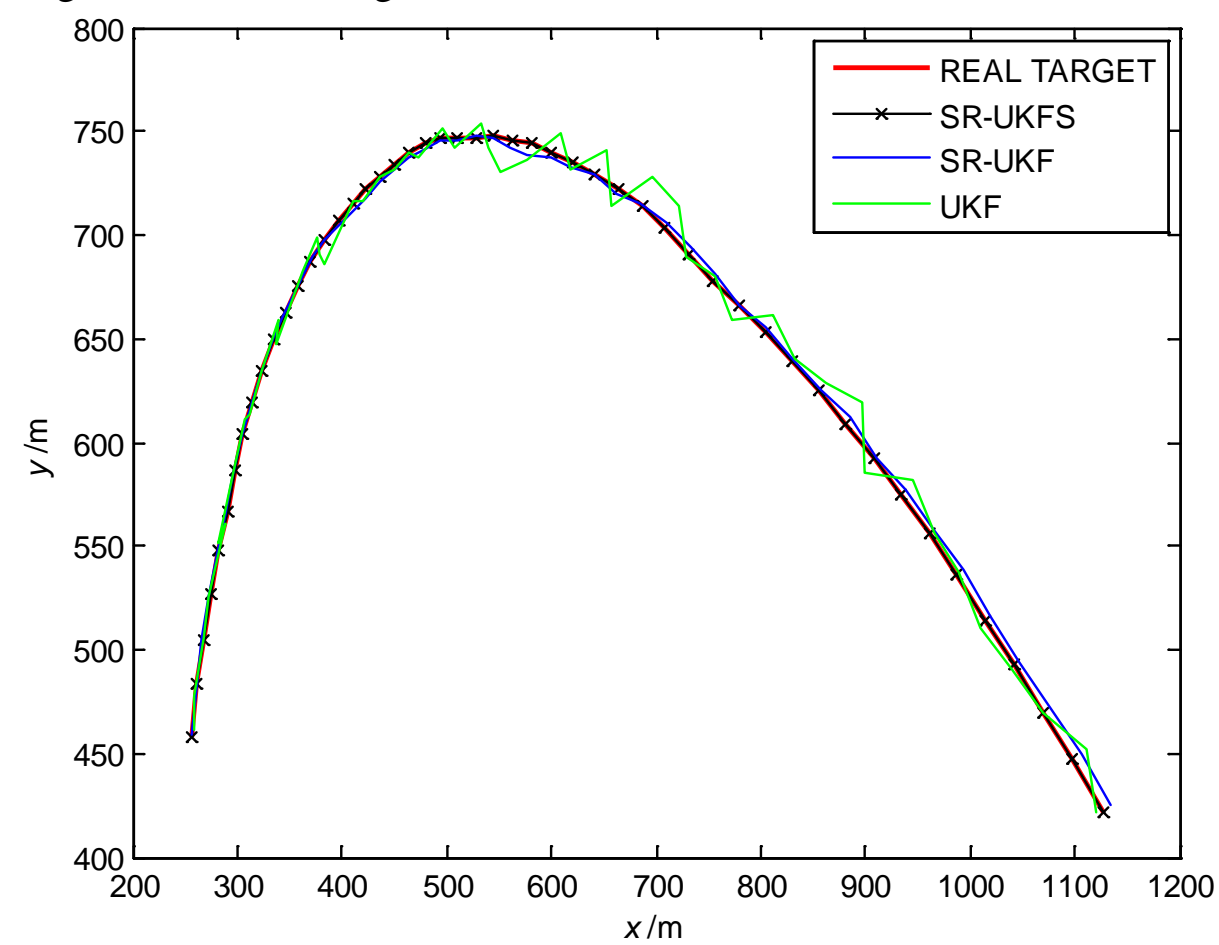

Fig.1 Comparison chart of the three kinds of algorithms in tracking

Tab.1 is the comparison of location and speed mean square after the filters of UKF,SR-UKF and SR-UKFS. The simulation results clearly show that UKF has the worst tracking accuracy, the stability of SR-UKF and SR-UKFS are better, and the tracking accuracy of SR-UKFS is better than SR-UKF.

Compared with the SR-UKF algorithm, the SR-UKFS algorithm can reduce nearly $59 \%$ of the position error and nearly $54 \%$ of the velocity error than the SR-UKF algorithm.

Tab.1 Comparison of estimation variances of the three kinds of algorithms

\begin{tabular}{ccccc}
\hline & $x / \mathrm{m}$ & $y / \mathrm{m}$ & $v_{x} /(\mathrm{m} / \mathrm{s})$ & $v_{y} /(\mathrm{m} / \mathrm{s})$ \\
\hline UKF & 1.8461 & 2.8102 & 0.0735 & 0.0564 \\
SR-UKF & 1.4127 & 1.0305 & 0.0598 & 0.0439 \\
SR-UKFS & 0.5807 & 0.9806 & 0.0275 & 0.0229 \\
\hline
\end{tabular}

\section{Conclusions}

In this paper, the author utilizes passive sonar to obtain the information of pitch angle and azimuth angle of underwater target and track underwater motion targets by combination with the SR-UKFS algorithm. Based on SR-UKFS algorithm, better filtering effect can be achieved to enhance the tracking accuracy.

The results of simulation show that SR-UKFS can be used in underwater motion target tracking, and the precision of filtering is significantly better than standard UKF and EKF algorithm. 


\section{Acknowledgements}

This research was supported by Zhejiang Provincial Natural Science Foundation of China under Grant No. LQ18E070004 and Grant No. LQ15F030007.

\section{References}

[1] Youfeng $\mathrm{Hu}$, Bingli Jiao. Passive underwater target motion analysis based upon bearing-elevation measurement in three dimensions [J]. Journal of System Simulation, 2003, 15(6): 776-784.

[2] Panlong Wu, Baobao Wang, Yadong Cai, et al. Single observer passive target tracking based on extended $H_{\infty}$ filter [J]. Journal of Chinese Inertial Technology, 2010,18(5): 591-599.

[3] Bo Wang, Demin Xu, Meng Shen. Underwater passive target motion analysis based on UKF [J]. Journal of Projectiles, Rockets, Missiles and Guidance, 2005, 25(2): 423-428.

[4] Panlong Wu, Baobao Wang, Cunhui Ji. Design and realization of short range defence radar target tracking system based on DSP/FPGA [J]. WSEAS Transactions on Systems, 2011, 10(11): 376-386.

[5] Baobao Wang, Lianzheng Zhang. Information fusion of airborne radar and ESM for maneuvering target tracking system based on IMM-BLUE [J]. WSEAS Transactions on Systems, 2014, 13(11): 699-707.

[6] Simon Julier, Jeffrey Uhlmann. A new method for the nonlinear transformation of means and covariances in filters and estimators [J]. IEEE Transactions on Automatic Control, 2000, 45(3): 477-482.

[7] Panlong Wu, Jianshou Kong. Under bearing-only target tracking based on Square-root UKF[J]. Journal of Nanjing University of Science and Technology (Natural Science Edition), 2009, 33(6): 751-755.

[8] Fan Zhang, Panlong Wu, Longmei Zhao. Improved SR-UKF algorithm for mobile robot tracking[J]. Journal of Computational Information System, 2012, 8(15): 6499-6506.

[9] Alfonso Farina. Target tracking with bearing-only measurements [J]. Signal Process, 1999, 78(1): 61-78.

[10] Chunlai Yu, Ronghui Zhan, Jianwei Wan. Research on robust UKF algorithm for single observer passive target tracking based on polar coordinates[J]. Journal of National University of Defense and Technology, 2008, 30(5): 73-79.

[11] S. Sadhu, S. Modndal, M. Srinivasan, et al. Sigma point Kalman filter for bearing only tracking [J]. Signal Processing,2006, 86(12): 3769-3777.

[12] Panlong Wu, Jiale Liu, Xingxiu Li. Maneuvering target tracking in clutter background based on improved interacting multiple-model [J]. Journal of Chinese Inertial Technology, 2015, 23(6): 755-762. 